\title{
Progreso técnico y productos deseados y no deseados en el Brasil: 1970-2012
}

\author{
Márcio Santetti, Adalmir Antônio Marquetti \\ y Henrique Morrone
}

\section{Resumen}

En el presente artículo se analiza el progreso técnico en la producción de productos deseados y no deseados en la economía brasileña en el período 1970-2012. Se considera que la combinación de los insumos trabajo, capital y energía genera un producto deseado, el producto interno bruto (PIB), y un producto no deseado, las emisiones de dióxido de carbono $\left(\mathrm{CO}_{2}\right)$. Los resultados revelan que, en el período analizado, la economía brasileña presentó un patrón de progreso técnico de sesgo marxista y ahorrador de energía. Sin embargo, se distinguen tres fases de cambio técnico: en la primera, entre 1970 y 1980, el progreso técnico tuvo un sesgo marxista, en la segunda, entre 1980 y 2003, hubo un estancamiento técnico y en la tercera, entre 2003 y 2012, se observó un patrón de progreso técnico neutral de Harrod.

\section{Palabras clave}

Crecimiento económico, cambio tecnológico, producción industrial, gas de efecto invernadero, análisis de insumo-producto, indicadores económicos, Brasil

\section{Clasificación JEL}

E01, E23, O33

\section{Autores}

Márcio Santetti es Doctorando en Economía de la Universidad de Utah, Estados Unidos, y tiene una Maestría en Economía del Desarrollo de la Pontificia Universidad Católica de Rio Grande do Sul (PUCRS), Brasil. Correo electrónico: santetti@gmail.com.

Adalmir Antônio Marquetti es Profesor Titular del Departamento de Economía y Postgrado en Economía del Desarrollo de la Pontificia Universidad Católica de Rio Grande do Sul (PUCRS), Brasil. Correo electrónico: aam@pucrs.br.

Henrique Morrone es Profesor Adjunto del Departamento de Economía de la Universidad Federal de Rio Grande do Sul (UFRGS), Brasil. Correo electrónico: hmorrone@hotmail.com. 


\section{Introducción}

El aumento exponencial de la producción en la sociedad capitalista fue posible gracias a la explotación de los recursos naturales, el empleo de trabajo humano, la expansión de la educación y la mayor utilización de máquinas y equipos que incorporan innovaciones técnicas ${ }^{1}$. El progreso técnico es fundamental para explicar el crecimiento económico. En el enfoque marxista clásico hay incentivos para la adopción de técnicas ahorradoras de trabajo y consumidoras de capital en la disputa por el valor agregado entre trabajadores y capitalistas. Las empresas adoptan cambios técnicos que reducen el costo de producción a los precios vigentes para obtener una ganancia superior a la de sus competidores. El progreso técnico asume la forma de mecanización, que se refleja en el aumento de la productividad del trabajo y la disminución de la productividad del capital. Con la acumulación de capital se intensifica cada vez más el uso de máquinas y equipos en el proceso productivo, que se traduce en un aumento de la relación capital-trabajo. De acuerdo con Foley y Michl (1999), este tipo de progreso técnico se denomina "de sesgo marxista".

La contrapartida del crecimiento económico vinculado con la mecanización son los efectos negativos en el medio ambiente. La mecanización requiere el empleo de energía para movilizar el capital físico productivo, que tiene efectos negativos en el medio ambiente. De acuerdo con Kümmel (1989), la percepción de que el avance industrial por medio de la mecanización acarreó efectos irreversibles en la sociedad y la naturaleza es relativamente reciente. Durante siglos, el flujo de generación de residuos se debió a la propagación de energía solar en la atmósfera, que se manifestaba en radiación de calor y no tenía consecuencias nocivas para el planeta. Sin embargo, la Revolución Industrial introdujo nuevas fuentes de difusión energética, sobre todo los combustibles fósiles como el carbón o el petróleo, que incrementaron los efectos negativos de la actividad económica en la naturaleza ${ }^{2}$. El uso intensivo de esos insumos energéticos viabilizó el crecimiento de la economía durante y después de la Revolución Industrial, en virtud de la escasa eficiencia de las fuentes energéticas predominantes hasta ese momento, como por ejemplo la madera (Harvey, 2006). Las emisiones de dióxido de carbono $^{3}$ derivadas de la utilización de esos combustibles serían uno de los factores responsables del calentamiento global (Stern, 2006; Foley, 2009) 45.

En consecuencia, el patrón de cambio técnico también conlleva hipótesis sobre el empleo de energía en el proceso productivo y la generación del producto no deseado. En el patrón de sesgo marxista se produce un aumento de la utilización de energía y la generación del producto no deseado, la productividad de la energía (la relación entre el producto deseado y la energía), la relación entre la energía y el trabajo y la relación entre la energía y la reserva de capital.

\footnotetext{
1 El sistema capitalista se basa en la búsqueda de beneficios cada vez mayores a partir de una acumulación sin límites. Con el tiempo, la dinámica capitalista engendró una creciente división entre el campo y la ciudad (Burkett, 2003) y los campos agrícolas dejaron de recibir flujos de habitantes de la ciudad para fertilizarlos. En otras palabras, la especialización productiva y la bipolarización campo-ciudad impidieron que la materia orgánica circulara de forma efectiva. Esa ruptura del ciclo de los nutrientes provocó el aumento de la contaminación en las ciudades (Foster, Clark y York, 2010). La escala de producción cada vez mayor y la aplicación de criterios empresariales al funcionamiento de las fincas agrícolas aceleraron la degradación del medio ambiente.

2 El petróleo se origina en materiales orgánicos (zooplancton y algas) fosilizados en eras pasadas como, por ejemplo, la Jurásica (169-144 millones de años atrás). Debido a su elevada densidad energética y fácil transporte y almacenamiento, el petróleo se convirtió en el principal insumo energético, de manera que el sistema mundial depende en gran medida de ese recurso natural (Li, 2014). El empleo de recursos naturales para alcanzar un crecimiento económico exponencial genera automáticamente un aumento de las emisiones de gases nocivos al medio ambiente, incluidos el dióxido de carbono $\left(\mathrm{CO}_{2}\right)$ y el metano $\left(\mathrm{CH}_{4}\right)$.

3 Los términos dióxido de carbono, gas carbónico y la fórmula $\mathrm{CO}_{2}$ se emplean de forma intercambiable a lo largo del texto.

4 Sobre la evolución del concepto de calentamiento global, véanse Arrhenius (1896), Callendar (1938) y Maslin (2004).

5 La producción de dióxido de carbono es uno de los principales ejemplos de generación de residuos en el capitalismo. Este gas representa el $77 \%$ de las emisiones mundiales de los componentes del efecto invernadero y 57 puntos porcentuales de esa cifra se deben a la quema de combustibles fósiles (IPCC, 2007). La contaminación es inherente a la producción capitalista y se configura como uno de los desdoblamientos de la creciente acumulación de capital.
} 
Siguiendo una perspectiva marxista clásica (Duménil y Levy, 1995; Foley y Michl, 1999; Marquetti y Pichardo, 2013), en el presente artículo se emplea un modelo que combina los insumos trabajo, capital y energía en la producción de un producto deseado, el producto interno bruto (PIB), y un producto no deseado, las emisiones de dióxido de carbono $\left(\mathrm{CO}_{2}\right)^{6}$. A partir de ese sistema, se investigan la producción de ambos productos y el progreso técnico en la economía brasileña en el período 1970-2012. La contribución del artículo consiste en proponer el empleo de energía como insumo y la producción de dióxido de carbono como un producto no deseado del proceso productivo.

Esto permite investigar el patrón de progreso técnico de una manera más precisa. Una de las principales hipótesis del trabajo es que, en el período citado, la economía brasileña presentó un patrón de progreso técnico de sesgo marxista, en particular en las fases de mayor crecimiento económico. Los resultados revelan un aumento en la producción del producto deseado a tasas superiores a las del producto no deseado y un patrón de progreso técnico caracterizado por el aumento de la productividad del trabajo, la reducción de la productividad del capital y la disminución de la tasa de ganancia en el Brasil entre 1970 y 2012.

El presente artículo se divide en cuatro secciones incluida esta introducción. En la segunda sección se describen el progreso técnico y la generación de productos deseados y no deseados mediante un análisis marxista clásico. En la tercera se analizan el PIB, las emisiones de $\mathrm{CO}_{2}$ y el patrón de progreso técnico en la economía brasileña entre 1970 y 2012. En la cuarta y última sección se presentan las consideraciones finales.

\section{Un sistema para estudiar la producción y el progreso técnico en la perspectiva marxista clásica}

A partir de las décadas de 1960 y 1970, se registraron cambios políticos y económicos relacionados con la contaminación y la explotación de los recursos naturales. La reconstrucción de los países centrales después de la Segunda Guerra Mundial y la expansión industrial estadounidense y soviética durante la Guerra Fría exigieron un mayor uso de energía y recursos naturales, que provocó altos niveles de contaminación. Los estudios pioneros de Carson (1962) y Leontief (1970) y el ascenso de la economía ecológica (Georgescu-Roegen, 1971; Daly, 1977) evidenciaron los elementos de compensación entre el crecimiento económico y los límites de los recursos naturales.

El proceso productivo comprende la transformación energética de insumos en mercancías finales, que conlleva intercambios de materia y energía con el medio ambiente. El proceso de transformación supone la generación de residuos, pues parte de la energía utilizada se disipa. Esos residuos tienen un impacto negativo en la naturaleza y sus ecosistemas.

El dióxido de carbono es uno de los principales contaminantes generados por la actividad económica. Este representa el $77 \%$ de las emisiones mundiales de gases de efecto invernadero. De esa cifra, 57 puntos porcentuales se deben a la quema de combustibles fósiles, mientras que 17 puntos porcentuales derivan de actividades de deforestación y de la descomposición de biomasa y los 3 puntos porcentuales restantes provienen de otras fuentes (IPCC, 2007). La acumulación de

\footnotetext{
6 Baran y Sweezy (1966) muestran que, además de las crecientes emisiones de $\mathrm{CO}_{2}$, la actividad económica capitalista produce una serie de desperdicios, que incluyen los gastos innecesarios en embalajes sofisticados y los costos cada vez mayores de la publicidad como formas de aumentar la demanda de los productos. Si bien son relevantes para explicar las ineficiencias del capitalismo y sus efectos en el medio ambiente, estas implicaciones escapan al alcance de este artículo.
} 
dióxido de carbono en la atmósfera es una consecuencia no intencional de la acción humana en la economía capitalista ${ }^{7}$.

En la década de 1960, se retomaron las líneas de investigación de los economistas clásicos y de Marx (Garegnani y Petri, 1989). En una de ellas se tiene en cuenta la tendencia decreciente de la tasa de ganancia para analizar las trayectorias a largo plazo de la economía mundial (Okishio, 1961; Morishima, 1973; Christiansen, 1976; Roemer, 1977).

El enfoque marxista clásico investiga el desarrollo capitalista sobre la base del conflicto entre capitalistas y trabajadores con respecto a la apropiación del excedente económico y los incentivos de la competencia en la adopción de técnicas para reducir los costos (Foley, 1998). La competencia impulsa a las empresas a adoptar cambios técnicos que reducen los costos de producción a fin de obtener una tasa de ganancia superior a la media. Marx describió ese proceso como el motor del cambio técnico en la producción capitalista. La expectativa de una rentabilidad superior a la media lleva a las empresas a adoptar cambios técnicos ahorradores de trabajo y consumidores de capital en el proceso productivo.

La creciente mecanización de la economía se evidencia en la expansión del uso de máquinas y equipos, recursos naturales y energía. El empleo de mano de obra aumenta cuando la acumulación de capital se incrementa por encima de la tasa de crecimiento de la productividad del trabajo. Esto se traduce en una mayor producción del producto deseado, que permite a los capitalistas obtener una tasa de ganancia, y en la generación de productos no deseados en forma de contaminación y desperdicio. No obstante, si los salarios aumentan de acuerdo con la productividad del trabajo, el proceso de mecanización puede reducir la tasa de ganancia.

La teoría marxista clásica de la reducción de la tasa de ganancia supone las siguientes tendencias a largo plazo para la economía capitalista:

i) aumento en la producción de los productos deseado y no deseado;

ii) crecimiento de la relación capital-trabajo;

iii) aumento de la productividad del trabajo y reducción de la productividad del capital, y

iv) reducción de la tasa de ganancia si se mantiene constante la distribución del ingreso.

Foley y Michl (1999) y Duménil y Levy (2003) desarrollaron modelos económicos para explicar las trayectorias de las economías capitalistas. Sobre la base de identidades contables y de la tradición marxista clásica, esos autores señalan que las sociedades presentan cambios técnicos de sesgo marxista a largo plazo (Pichardo, 2007). Si la energía utilizada en el proceso productivo se basa en combustibles fósiles, aumentará la emisión de gas carbónico.

Para el análisis de las tendencias de crecimiento económico y cambio técnico, se considera una economía que produce un producto deseado, $X$, y un producto no deseado, $B$. El producto deseado está representado por el PIB, medido en reales de 1995 sobre la base de IBGE (1990) e IBGE (2003) para los años 1970-1985 y de IBGE (2010) para los años 1995-2008. Para el período 2008-2012 se utilizó IPEA (2016). En el presente trabajo, $B$ está representada por las emisiones de dióxido de carbono $\left(\mathrm{CO}_{2}\right)$. Los datos relativos a la emisión de $\mathrm{CO}_{2}$, en kilogramos $(\mathrm{kg})$, para el período 1970-2008, se tomaron de Boden, Marland y Andres (2016).

Al término de cada período, una parte de la reserva de capital se deprecia. $K$ - $D$ se refiere al monto de capital restante al final del período de producción. La tasa de depreciación es la razón entre

\footnotetext{
7 Desde la prehistoria, se verifican ciclos de alta y baja concentración de dióxido de carbono en la atmósfera (Vicente, 2014). Sin la influencia del hombre, los flujos naturales de gas carbónico siguen un patrón cíclico. Sin embargo, a partir de 1750, ese comportamiento cesa y la concentración atmosférica de $\mathrm{CO}_{2}$ aumenta considerablemente. Esto coincide con el inicio de la industrialización, posibilitada por el uso de combustibles fósiles como fuentes energéticas. La deforestación de bosques para promover actividades agrícolas también contribuyó al incremento de las emisiones (Vitousek y otros, 1997).
} 
la depreciación y la reserva de capital $(\delta=D / K)^{8}$. En el cuadro 1 se resume el proceso de producción de los productos deseado y no deseado, a partir de los insumos capital, trabajo y energía.

Cuadro 1

Relación insumo-producto de la generación de los productos deseado y no deseado

\begin{tabular}{cccccc}
\hline & Insumos & \multicolumn{3}{c}{ Productos } \\
\hline Capital & Trabajo & Energía & Deseado & No deseado & Capital \\
\hline$K$ & $N$ & $E$ & $X$ & $B$ & $K-D$ \\
\hline
\end{tabular}

Fuente: Elaboración propia.

El proceso productivo de una economía puede representarse mediante una función de producción. Esa función indica la manera en que los insumos se combinan para generar una mercancía final. Las ecuaciones (1) y (2) ilustran las funciones de producción, de tipo Leontief, para $X$ y $B$, respectivamente.

$$
\begin{gathered}
X=\min (\rho K, x N, e E) \\
B=\min (a K, b N, c E)
\end{gathered}
$$

$K$ representa la reserva neta de capital fijo. Esta se estimó con el método del inventario permanente y se midió en reales de 1995 (Marquetti y Porsse, 2014). Los datos relativos a la formación bruta de capital fijo se obtuvieron en IBGE (2003) (período 1970-1985), IBGE (2010) (período 1995-2008) e IPEA (2016) (período 2009-2012). $N$ es el número de trabajadores. Las fuentes de datos son IBGE (2003), para el período 1990-1995, e IBGE (2010 y 2015) para el período 1995-2012. Para los demás años se utilizaron los censos nacionales de 1970, 1975, 1980 y 1985 y la base de datos de Heston, Summers y Aten (2006). $E$ es la oferta de energía. Los datos relativos a la oferta interna de energía en el período 1970-2012, expresados en toneladas equivalentes de petróleo (TEP), se obtuvieron a partir de las series históricas completas del Balance Energético Nacional, elaborado por la Empresa de Investigación Energética del Ministerio de Minas y Energía (MME, 2014). $x=X / N$ es la productividad del trabajo, medida en reales de 1995 por trabajador; $\rho=X / K$ es la productividad del capital, que es un número puro como una tasa de interés, medida en porcentaje por año, y $e=X / E$ es la productividad de la energía, la razón entre el producto deseado y la oferta de energía, medida en reales de 1995 por TEP. $a=B / K$ es la emisión de $\mathrm{CO}_{2}$ por unidad de capital, medida en toneladas por reales de 1995; $b=B / N$ es la emisión de $\mathrm{CO}_{2}$ por unidad de trabajo, medida en toneladas por trabajador, y $c=B / E$ es la emisión de $\mathrm{CO}_{2}$ por unidad de energía, medida en toneladas por TEP. Por último, $o=X / B$ es la razón entre el PIB y el $\mathrm{CO}_{2}$, medida en reales de 1995 por tonelada.

La tecnología está representada por el conjunto de técnicas productivas disponibles en una economía en un período determinado. Una técnica de producción puede describirse mediante variables técnicas y mediante variables de intensidad de emisión. Las primeras están representadas por los parámetros $(x, \rho, e)$, ligados a la producción del producto deseado, y las segundas, por los parámetros $(a, b, c)$, que se refieren a la producción del producto no deseado. De acuerdo con Foley y Michl (1999), una técnica de producción presenta tres características con respecto al proceso productivo: i) las cantidades de capital y energía necesarias para proporcionar una unidad de trabajo, las relaciones capital-trabajo $(k=K / N)$ y energía-trabajo $(\varepsilon=E / N)$; ii) la cantidad de productos deseados y no deseados generados al final del período por trabajador, y iii) la cantidad de reserva de capital que se deprecia en un período de producción. En el cuadro 2 se presentan los coeficientes insumo-producto.

\footnotetext{
8 Cabe señalar que los símbolos de las variables y de los parámetros seleccionados se fundan en una literatura bastante consolidada sobre crecimiento económico, cuya base principal es Foley y Michl (1999). Marquetti y Porsse (2014) también emplean las mismas variables en un análisis sobre América Latina.
} 
Cuadro 2

Coeficientes insumo-producto de la generación de los productos deseado y no deseado

\begin{tabular}{cccccc}
\hline & Insumos & \multicolumn{3}{c}{ Productos } \\
\hline Capital & Energía & Trabajo & Deseado & No deseado & Capital \\
\hline$k$ & $e$ & 1 & $x$ & $b$ & $(1-\delta) k$ \\
\hline
\end{tabular}

Fuente: Elaboración propia.

El cambio técnico consiste en la variación de por lo menos uno de los parámetros $(x, \rho, e)$ y $(a, b, c)$ a lo largo del tiempo y puede representarse mediante tasas de crecimiento. Por ejemplo, la tasa de crecimiento de la productividad del trabajo se calcula por $g_{x}=\Delta x / x$, donde $\Delta$ representa la variación del parámetro entre dos períodos. De esa forma, $g_{\rho}=\Delta \rho / \rho$ es la tasa de crecimiento de la productividad del capital, $g_{e}=\Delta e / e$ es la tasa de crecimiento de la productividad de la energía, $g_{a}=\Delta a / a$ es la tasa de crecimiento de las emisiones por unidad de capital, $g_{b}=\Delta b / b$ es la tasa de crecimiento de las emisiones por unidad de trabajo, $g_{c}=\Delta c / c$ es la tasa de crecimiento de las emisiones por unidad de energía y $g_{o}=\Delta o / o$ es la tasa de crecimiento de la razón entre el producto deseado y el no deseado. El cambio técnico se considera neutral cuando su adopción no supone variaciones en la distribución del ingreso (Jones, 1979). Por ejemplo, el progreso técnico neutral de Harrod corresponde a un aumento de la tasa de crecimiento de la productividad del trabajo $\left(g_{x}>0\right)$, mientras se mantiene constante la tasa de crecimiento de la productividad del capital $\left(g_{\rho}=0\right)$. El progreso técnico neutral de Solow consiste en el aumento de la tasa de crecimiento de la productividad del capital $\left(g_{\rho}>0\right)$, mientras permanece constante la tasa de crecimiento de la productividad del trabajo $\left(g_{x}=0\right)$. El progreso técnico neutral de Hicks es ahorrador en todos los insumos y supone aumentos equivalentes en las respectivas tasas de crecimiento de las productividades $\left(g_{x}=g_{\rho}=g_{e}\right)$.

De acuerdo con Marquetti (2003), a lo largo del siglo XX predominaron los cambios técnicos ahorradores de trabajo y consumidores de capital. En otras palabras, aumentó la tasa de crecimiento de la productividad del trabajo $\left(g_{x}>0\right)$ y se redujo la tasa de crecimiento de la productividad del capital $\left(g_{\rho}<0\right)$. El cambio técnico observado es coherente con el análisis de Marx (1991) sobre el progreso técnico en el modo de producción capitalista. La creciente mecanización de las economías exige una menor cantidad de trabajo y una mayor cantidad de capital para generar una unidad de producto, de manera que aumenta la productividad del trabajo y se reduce la productividad del capital. Este patrón de progreso técnico se denomina de sesgo marxista (Foley y Michl, 1999; Marquetti, 2003; Pichardo, 2007).

\section{Crecimiento, progreso técnico e intensidad de emisión en el Brasil: 1970-2012}

En esta sección se investigan el crecimiento económico brasileño, el patrón de progreso técnico y los indicadores de intensidad de emisión en el período estudiado. Entre 1970 y 2012, la economía brasileña creció a una tasa del 4,13\% anual, mientras la tasa de crecimiento anual de las emisiones de dióxido de carbono fue del 3,87\%. Sin embargo, como se puede observar en el gráfico 1, es posible distinguir tres períodos de crecimiento en el Brasil. Entre 1970 y 1980, el producto deseado creció un 8,27\% -impulsado por el "milagro económico" y el II Plan Nacional de Desarrollo (II PND) - y la emisión de gas carbónico se incrementó un 6,91\%. Entre 1980 y 2003, el PIB brasileño y la emisión de gas carbónico crecieron a tasas similares, del 2,37\% y el 2,40\% al año, respectivamente. En la década perdida se registró un bajo crecimiento, acompañado por elevadas tasas de inflación. Con la adopción del neoliberalismo en la década de 1990, la economía brasileña continuó su trayectoria de bajo crecimiento. Entre 2003 y 2012, la adopción de una política que combinaba elementos del 
neoliberalismo con el desarrollismo se tradujo en un mayor dinamismo económico. Las tasas de crecimiento del PIB y de la emisión de gas carbónico fueron similares a las medias observadas en el período en su conjunto.

\section{Gráfico 1}

Brasil: producción de los productos deseado y no deseado y uso de los insumos trabajo, capital y energía, 1970-2012

A. Producto interno bruto (PIB)

(En millones de reales de 1995)

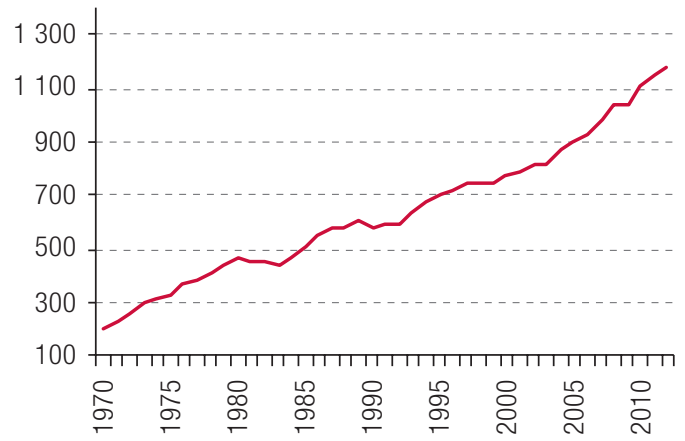

C. Reserva de capital

(En millones de reales de 1995)

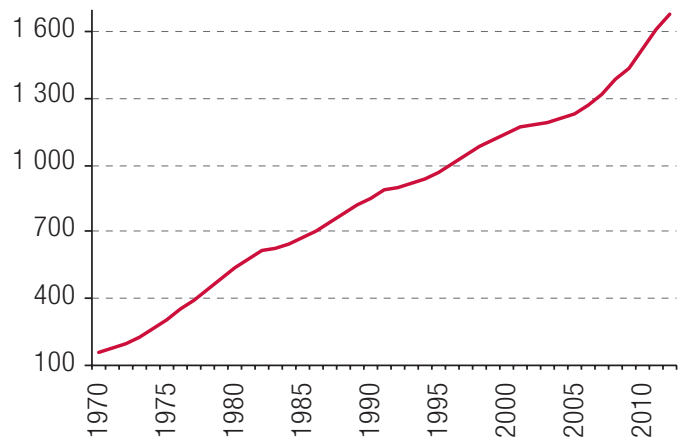

E. Oferta de energía

(En millones de toneladas equivalentes de petróleo)

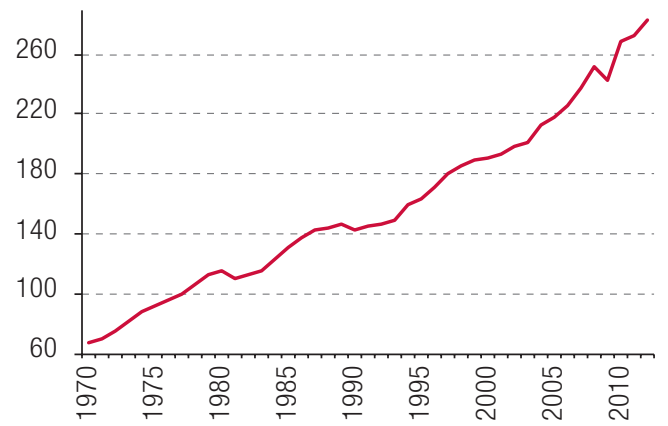

B. Emisiones de dióxido de carbono

(En trillones de kilogramos)

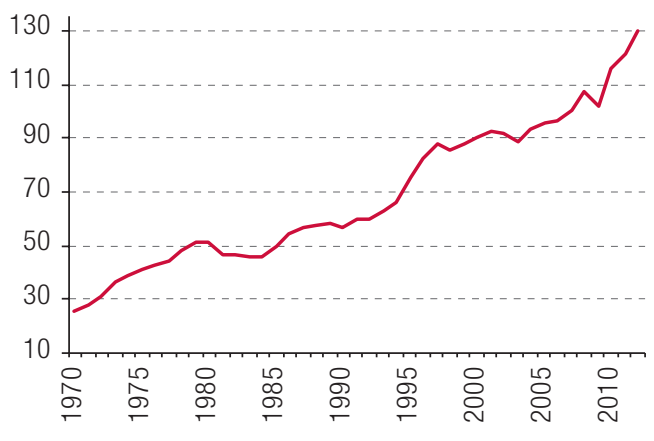

D. Número de trabajadores

(En millones de personas)

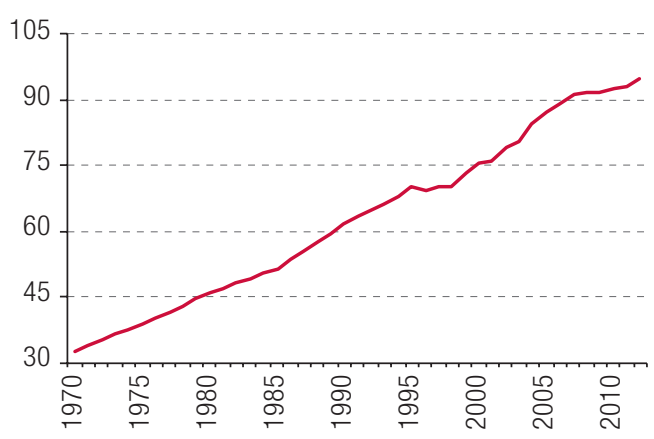

Fuente: Elaboración propia. 
Entre 1970 y 2012, los insumos de capital, trabajo y energía crecieron a una tasa media del $5,66 \%$, el 2,53\% y el 3,43\% al año, respectivamente. A partir del gráfico 1, también se observan modificaciones en el incremento de la utilización de los insumos que son coherentes con las tres fases de la economía brasileña. La tasa de crecimiento de la reserva de capital se redujo del 12,39\% en la década de 1970 al 3,45\% entre 1980 y 2003.

La rápida acumulación de capital en la década de 1970 se debió a la intensificación del proceso de sustitución de importaciones en el marco del II PND. Gran parte de las inversiones se financió mediante préstamos internacionales. De acuerdo con Marquetti y Porsse (2014), la disminución de la rentabilidad y el incremento de la tasa de interés internacional determinaron la reducción de la tasa de acumulación de capital en la década de 1980. Esa tendencia se mantuvo entre 1989 y 2003. A fines de los años ochenta, comenzó el proceso de desindustrialización de la economía brasileña con la pérdida de participación de la industria en el PIB y las exportaciones ${ }^{9}$. La tasa de acumulación de capital se recuperó levemente entre 2003 y 2012, cuando la reserva de capital aumentó a una media del 3,83\% al año.

Si bien el número de trabajadores empleados también creció más rápidamente en la década de 1970, la disminución de esta tasa de crecimiento en el período 1980-2003 no fue tan acentuada como en el caso de los demás insumos. El empleo en el sector de servicios aumentó con mayor velocidad que en el sector industrial. Esto puede ilustrarse mediante un análisis reciente de los datos del cuadro de recursos y usos publicado por el Instituto Brasileño de Geografía y Estadística (IBGE). En 1996, el sector de servicios representaba el 40,6\% de los empleados de la economía. En 2009, esa participación había aumentado al 45,6\%. La industria registró un crecimiento más modesto, al pasar del 18,9\% en 1996 al 19,6\% en 2009 (Jacinto y Ribeiro, 2015). Entre 2003 y 2012, el incremento del número de trabajadores fue menor que en las fases anteriores. La crisis de 2008 puede haber tenido un efecto mayor en la expansión del número de trabajadores que en los otros insumos.

En la década de 1970, hubo un rápido aumento en la producción de energía, que creció a una tasa media del 5,38\% anual. Entre 1980 y 2003, esa tasa de crecimiento disminuyó al 2,44\% anual. En el período entre 2003 y 2012, se reanudó el crecimiento de la oferta de energía en el país. Estos movimientos indican una relación entre las fases de crecimiento de la economía brasileña y la ampliación de la oferta de energía.

Existe una particularidad con respecto a las fuentes energéticas en el Brasil. En 1970, el 58,4\% de la oferta interna de energía del país se basaba en fuentes renovables. La economía brasileña diversificó su matriz energética. Por ejemplo, la leña y el carbón vegetal, fuentes muy contaminantes que representaban el $48 \%$ de la matriz energética en 1970, disminuyeron al 12\% en 2008. Nuevas fuentes de energía, como los derivados de la caña de azúcar y la energía hidráulica, aumentaron su participación en la oferta interna. No obstante, la dependencia del petróleo disminuyó poco, del 38\% en 1970 al $36,6 \%$ en 2008. En ese último año, el 46,1\% de la energía correspondía a fuentes no renovables, como petróleo y derivados, gas natural, carbón mineral y uranio (MME, 2014). La participación de las fuentes renovables en la matriz energética del resto del mundo era del 12,7\% (MME, 2007).

En un contexto de producción de productos deseados y no deseados, el progreso técnico puede analizarse mediante la evolución de variables técnicas $(x, \rho, e, k, \varepsilon)$ y de variables relativas a la intensidad de emisión del producto no deseado $(a, b, c, o)$. La dinámica del progreso técnico fue influenciada por el crecimiento de la economía brasileña entre 1970 y 2012.

En el gráfico 2 se muestran la evolución de las variables técnicas y la tasa de ganancia y la razón entre ganancias y reserva de capital entre 1970 y $2012^{10}$. En ese período aumentó la productividad

\footnotetext{
9 Sobre la desindustrialización de la economía brasileña, véanse Furtado y Carvalho (2005) y Feijó y Lamonica (2012).

10 Véase un análisis de la tasa de ganancia en el Brasil y sus relaciones con las variables técnicas en el período estudiado en Marquetti y Porsse (2014). La unidad de medida de la tasa de ganancia es el porcentaje por año.
} 
del trabajo (véase el gráfico 2A), disminuyó la productividad del capital (véase el gráfico 2B), creció moderadamente la productividad de la energía (véase el gráfico $2 \mathrm{C}$ ), aumentó la razón capital-trabajo (véase el gráfico 2D), creció la razón energía-trabajo (véase el gráfico 2E) y disminuyó la tasa de ganancia (véase el gráfico 2F). El patrón de cambio técnico fue coherente con el patrón de sesgo marxista.

\section{Gráfico 2}

Brasil: patrones de cambio técnico y tasa de ganancia, 1970-2012

A. Productividad del trabajo

(En reales de 1995 por trabajador)

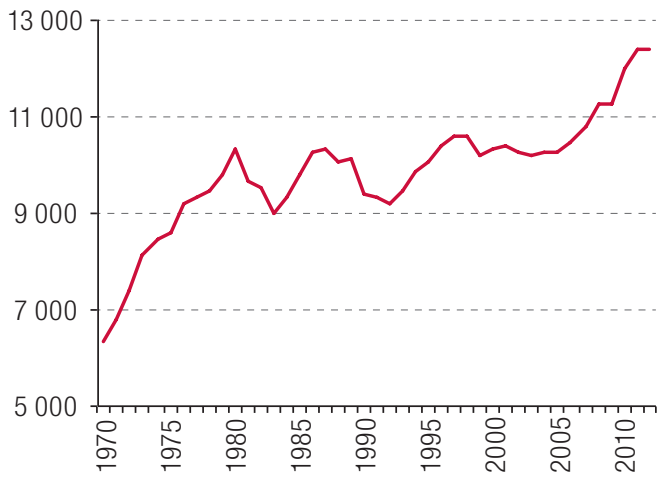

C. Productividad de la energía

(En reales de 1995 por tonelada equivalente de petróleo)

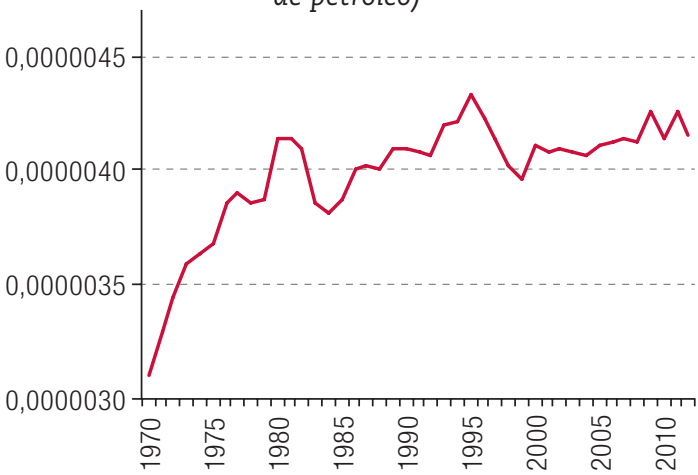

E. Relación energía-trabajo

(En toneladas equivalentes de petróleo por trabajador)

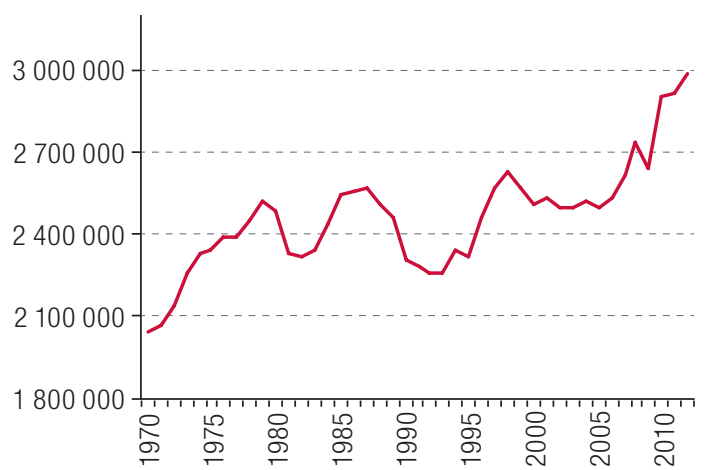

B. Productividad del capital

(En porcentajes)

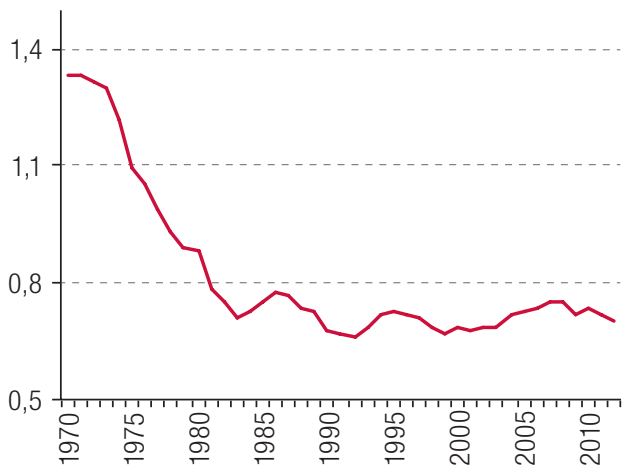

D. Razón capital-trabajo

(En reales de 1995 por trabajador)

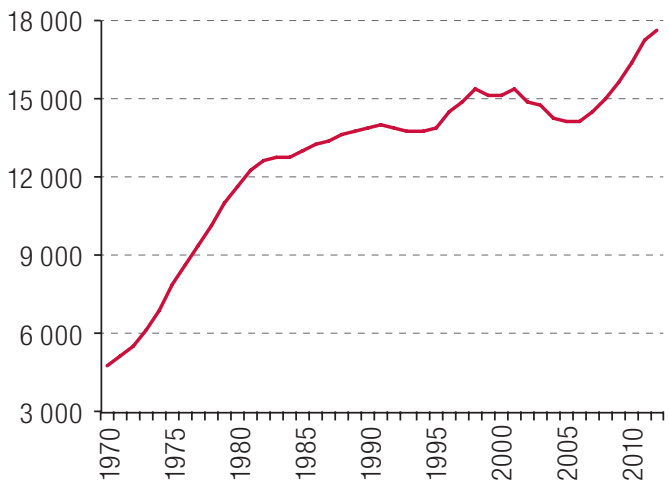

F. Tasa de ganancia

(En porcentajes)

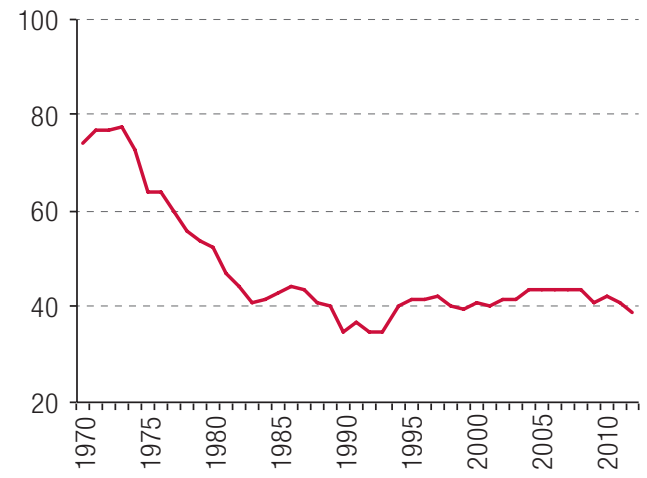

Fuente: Elaboración propia. 
Sin embargo, en la evolución de las variables de cambio técnico y el patrón de progreso técnico se distinguen tres fases coherentes con el crecimiento de la economía brasileña. En la primera, entre 1970 y 1980, hubo un acentuado proceso de mecanización de la economía brasileña y, en promedio, la productividad del trabajo creció un 4,88\% al año, la productividad del capital disminuyó un 4,1\% al año y la productividad de la energía aumentó un 2,89\% al año (véase el cuadro 3). En esa fase, las tasas anuales de crecimiento de las tres variables técnicas mencionadas fueron estadísticamente diferentes de cero al 5\% de significación, y el patrón de progreso técnico fue de sesgo marxista.

\section{Cuadro 3}

Brasil: tasas anuales de crecimiento del producto interno bruto (PIB), las emisiones de dióxido de carbono $\left(\mathrm{CO}_{2}\right)$, los insumos y las variables técnicas y de intensidad de emisión de $\mathrm{CO}_{2}, 1970-2012$

(En porcentajes)

\begin{tabular}{|c|c|c|c|c|c|c|c|c|c|c|c|c|c|c|}
\hline Período & $g X$ & $\mathrm{gB}$ & gK & $\mathrm{gN}$ & $\mathrm{gE}$ & $g x$ & $g \rho$ & ge & gk & $g \varepsilon$ & ga & $g b$ & gc & go \\
\hline $1970-2012$ & 4,13 & 3,87 & 5,66 & 2,53 & 3,43 & 1,60 & $-1,53$ & 0,70 & 3,13 & 0,90 & $-1,79$ & 1,34 & 0,44 & 0,26 \\
\hline $1970-1980$ & 8,27 & 6,91 & 12,39 & 3,40 & 5,38 & 4,88 & $-4,11$ & 2,89 & 8,99 & 1,99 & $-5,48$ & 3,51 & 1,53 & 1,37 \\
\hline $1980-2003$ & 2,37 & 2,40 & 3,45 & 2,43 & 2,44 & $-0,06$ & $-0,58$ & $-0,07$ & 0,94 & 0,01 & $-1,05$ & $-0,03$ & $-0,04$ & $-0,03$ \\
\hline 1980-1989 & 2,62 & 1,49 & 4,72 & 2,88 & 2,75 & $-0,25$ & $-2,10$ & $-0,12$ & 1,85 & $-0,13$ & $-3,23$ & $-1,38$ & $-1,25$ & 1,13 \\
\hline 1989-2003 & 2,21 & 2,98 & 2,63 & 2,14 & 2,24 & 0,07 & $-0,42$ & $-0,03$ & 0,48 & 0,10 & 0,35 & 0,83 & 0,74 & $-0,77$ \\
\hline 2003-2012 & 4,02 & 4,27 & 3,83 & 2,03 & 3,82 & 1,99 & 0,19 & 0,20 & 1,80 & 1,79 & 0,44 & 2,24 & 0,45 & $-0,25$ \\
\hline
\end{tabular}

Fuente: Elaboración propia.

En la segunda fase, entre el inicio de la década de 1980 y 2003, hubo un estancamiento técnico en la economía brasileña y las variables técnicas y de intensidad de emisión mostraron movimientos cíclicos. Como se observa en el cuadro 3, los valores están muy cerca de cero en todas las variables técnicas y de intensidad de emisión de gas carbónico. Las tasas de crecimiento anual de la productividad del trabajo, la productividad del capital y la productividad energética no son estadísticamente diferentes de cero en el período entre 1980 y 2003. Por lo tanto, esa fase corresponde a un estancamiento técnico.

Entre 2003 y 2012 se registra una nueva fase: en promedio, la productividad del trabajo creció un 1,99\% al año, la productividad del capital aumentó un 0,19\% y la productividad de la energía creció un 0,20\% al año. Los resultados de las pruebas estadísticas revelan que las tasas anuales de crecimiento de la productividad del trabajo fueron estadísticamente diferentes de cero, a diferencia de la productividad del capital y de la energía. La economía brasileña presentó un patrón de progreso técnico neutral de Harrod (Foley y Michl, 1999).

Después de 2003, el Gobierno intensificó sus esfuerzos para promover el crecimiento económico. En un contexto externo favorable, debido al aumento de los precios de los productos básicos, se amplió el margen para la implementación de políticas macroeconómicas desarrollistas. Pese a los efectos negativos de la crisis de las hipotecas de alto riesgo en la economía, el Gobierno logró promover una rápida recuperación por medio de políticas anticíclicas.

En ese contexto de aumento de la productividad del trabajo y del ritmo de actividad económica, el incremento de la productividad energética es fundamental para mitigar los efectos del crecimiento económico en la emisión de contaminantes (Von Arnim y Rada, 2011). Así, la misma oferta de energía propicia una mayor producción del producto deseado o el mantenimiento del ritmo de crecimiento con menos energía. 
El crecimiento de la productividad del trabajo puede descomponerse ex post en la suma de dos componentes: la intensidad energética y la productividad energética (Von Arnim y Rada, 2011) ${ }^{1112}$. En el Brasil, ambos componentes aumentaron en la década de 1970. La productividad energética contribuyó en un 59\% al crecimiento de la productividad del trabajo en la primera fase (entre 1970 y 1980). En la segunda (entre 1980 y 2003), las variables presentaron tasas próximas a cero, y el período se caracterizó por un marcado estancamiento económico. En la tercera fase (entre 2003 y 2012), el crecimiento se relacionó sobre todo con la intensidad energética, que contribuyó en un 90\% al crecimiento de la productividad del trabajo. Este crecimiento, incentivado principalmente por la intensidad energética, es similar al observado en países con dificultades para controlar las emisiones de $\mathrm{CO}_{2}$ (Von Arnim y Rada, 2011).

Históricamente, el crecimiento económico de los países en desarrollo está vinculado con el aumento de la intensidad energética, que ocasiona el deterioro del medio ambiente (Taylor, 2008). En el período objeto de este análisis, de 1970 a 2012, la intensidad energética explicó el 57\% del crecimiento de la productividad del trabajo, mientras que el resto fue explicado por la otra variable.

En el gráfico 3 se presenta la evolución de las variables de intensidad de emisión de $\mathrm{CO}_{2}$ en el período 1970-2012. En promedio, se verificó una reducción del 1,79\% al año de las emisiones por unidad de capital (véase el gráfico 3A), un aumento del 1,34\% al año de las emisiones por trabajador (véase el gráfico 3B), un crecimiento del $0,44 \%$ al año de las emisiones por unidad de energía (véase el gráfico 3C) y un leve aumento del 0,26\% al año de la razón entre los productos deseado y no deseado (véase el gráfico 3D). No obstante, las fases de crecimiento y de cambio técnico no son tan nítidas como en el caso del crecimiento de los productos y de las variables técnicas.

Gráfico 3

Brasil: patrones de cambio en la intensidad de emisión del producto no deseado, 1970-2012

A. Emisiones por unidad de capital

(En toneladas por reales de 1995)

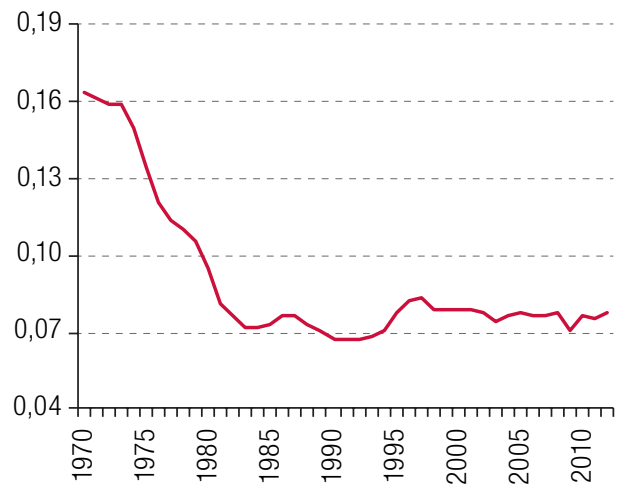

B. Emisiones de $\mathrm{CO}_{2}$ por trabajador (En toneladas)

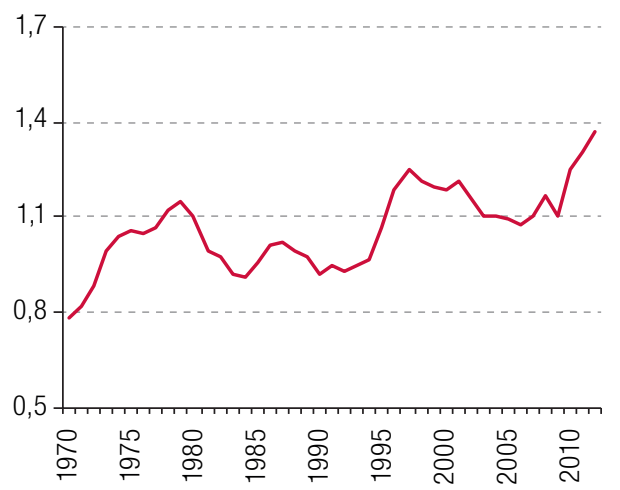

${ }^{11}$ La productividad del trabajo puede formularse de la siguiente manera: $x=e \quad \varepsilon$, o $\mathrm{X} / \mathrm{N}=(\mathrm{X} / \mathrm{E})(\mathrm{E} / \mathrm{N})$. Es posible realizar la diferenciación en logaritmos y concluir que $g x=g e+g \varepsilon$. Divisia (1926), Ocampo, Rada y Taylor (2009) y Von Arnim y Rada (2011) son algunos de los autores que realizaron la descomposición del crecimiento.

12 Una forma alternativa de descomponer la productividad del trabajo consiste en el empleo de la siguiente ecuación: $X / N=(X / K)$ $(\mathrm{K} / \mathrm{N})=(\rho)(\mathrm{K})$. Al diferenciar y aplicar logaritmos a esa expresión matemática, se verifica que $g x=g \rho+g k$. Si se iguala ese resultado con la descomposición de la nota al pie de página número 11, se puede afirmar que $g x=g e+g \varepsilon=g \rho+g k$. Así, si E/K es constante, se obtiene que $g \varepsilon=g k$. 
Gráfico 3 (conclusión)

C. Emisiones de $\mathrm{CO}_{2}$ por unidad de energía

(En toneladas de $\mathrm{CO}_{2}$ por tonelada equivalente de petróleo)

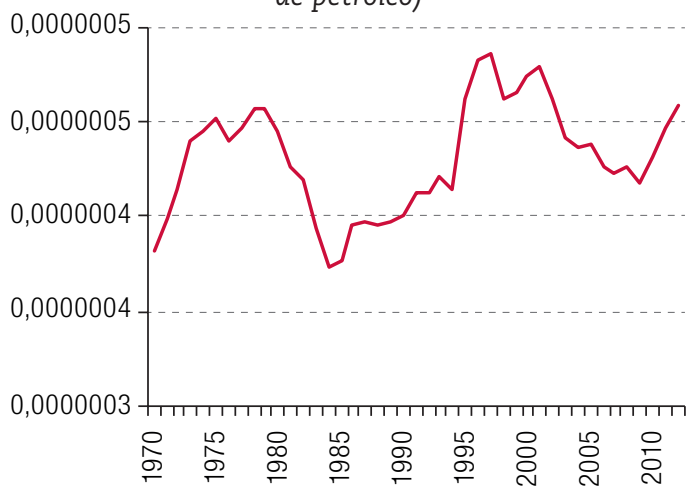

D. Razón producto deseado-producto no deseado (En reales de 1995 por tonelada)

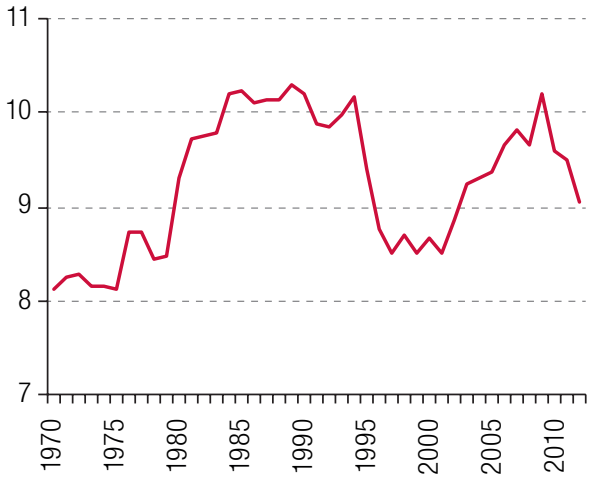

Fuente: Elaboración propia.

En promedio, las emisiones por unidad de capital disminuyeron un 5,48\% al año entre 1970 y 1980. Entre el inicio de la década de 1980 y 2012, estas oscilaron moderadamente. Esto puede explicarse por la reducción del crecimiento de las emisiones de $\mathrm{CO}_{2}$ debido a la moderación del uso de derivados de petróleo en la producción y a la menor acumulación de capital. En 2012, el nivel de emisiones por unidad de capital fue similar al del inicio de la década de 1980. Los resultados de las pruebas estadísticas muestran que la tasa de crecimiento de las emisiones por unidad de capital fueron negativas y significativamente diferentes de cero al $5 \%$ de significación en el período 1970-1980. Sin embargo, las pruebas revelan que la tasa de crecimiento de esa variable no fue significativamente diferente de cero en los períodos 1980-2003 y 2003-2012.

Entre 1970 y 1980, las emisiones de $\mathrm{CO}_{2}$ por trabajador aumentaron en promedio un 3,51\% al año, la mayor tasa de crecimiento de todo el período analizado. Las emisiones por trabajador presentaron un comportamiento cíclico, aumentando cuando había mayor crecimiento y disminuyendo cuando este se reducía. Así, estas disminuyeron entre 1980 y 1984, se mantuvieron estables hasta 1994 y aumentaron hasta 1997, cuando comenzó una nueva tendencia decreciente que duró hasta 2003. Entre 2003 y 2012, las emisiones de $\mathrm{CO}_{2}$ por trabajador aumentaron en promedio un 2,24\% al año, aumento concentrado en el final del período. Las pruebas estadísticas revelan que las tasas de crecimiento de las emisiones de $\mathrm{CO}_{2}$ por trabajador fueron significativamente diferentes de cero al $5 \%$ de significación en la primera fase entre 1970 y 1980. En las dos fases siguientes, las pruebas no fueron significativas estadísticamente.

En el gráfico $3 \mathrm{C}$ se muestran las emisiones de $\mathrm{CO}_{2}$ por unidad de energía, que en promedio aumentaron un $0,44 \%$ al año en el período de referencia. El movimiento de esa variable fue afectado por los ciclos económicos y los precios del petróleo; aumentó rápidamente en los períodos de auge y, aparentemente, se redujo en los períodos de aumento del precio de los combustibles fósiles. En 2008, las emisiones por unidad de energía supusieron 425 kilogramos por tonelada equivalente de petróleo, reflejando el aumento de la participación de las fuentes no renovables en la matriz energética. En 1970, ese valor era 381 kg/TEP. Después de disminuir en 2008 y 2009, el crecimiento de esa variable se aceleró. Los resultados de las pruebas estadísticas muestran que las tasas anuales de crecimiento de las emisiones de $\mathrm{CO}_{2}$ por unidad de energía no fueron significativamente diferentes de cero al $5 \%$ en ninguna de las tres fases analizadas.

Por último, la razón entre el producto deseado y el producto no deseado (véase el gráfico 3D) aumentó un $0,26 \%$ al año entre 1970 y 2012. Una vez más, la variable presentó un comportamiento cíclico, si bien no hay una relación clara con el ciclo económico. 
La pérdida de dinamismo del progreso técnico a partir de 1980 derivó de factores internos y externos de la economía brasileira. El principal factor interno fue la reducción de la tasa de acumulación de capital debido a la disminución de la tasa de ganancia. Con respecto a los factores externos, el aumento de las tasas de interés internacionales y los pagos de la deuda externa redujeron la capacidad de inversión, imposibilitando el mantenimiento de tasas de crecimiento elevadas.

Además, las innovaciones de la quinta revolución tecnológica (era de la información y las telecomunicaciones) tuvieron pocas repercusiones económicas en las décadas de 1980 y 1990. En el caso del Brasil, esas innovaciones no provocaron cambios estructurales significativos. Pérez (2002) argumenta que los efectos económicos de esas innovaciones fueron menores que los observados en las revoluciones tecnológicas anteriores.

Por último, en el cuadro 3 se presentan las tasas anuales de crecimiento de los dos productos, de los insumos trabajo, capital y energía y de las variables técnicas y de intensidad de emisión de $\mathrm{CO}_{2}$ en el período 1970-2012 y en diferentes fases del crecimiento económico brasileño. Las mayores tasas de crecimiento se registraron entre 1970 y 1980, cuando el patrón de progreso técnico adquirió el sesgo marxista. Durante la crisis del modelo de industrialización por sustitución de importaciones en los años ochenta y el neoliberalismo, entre el final de la década de 1980 y 2003, hubo un estancamiento técnico en el Brasil.

El crecimiento se reanudó en cierta medida después de 2003, con incrementos en el PIB, la generación de $\mathrm{CO}_{2}$ y el uso de los insumos examinados. Las variables técnicas y de intensidad de emisión muestran un patrón neutral de Harrod. En el período en su conjunto (1970-2012), el patrón de progreso técnico fue de sesgo marxista y ahorrador de energía.

\section{Consideraciones finales}

En este artículo se investigaron el progreso técnico y la producción de productos deseados y no deseados en la economía brasileña en el período 1970-2012. Se consideró que la economía produce un producto deseado, el PIB, y un producto no deseado, las emisiones de dióxido de carbono. Se observó el comportamiento de parámetros técnicos y de intensidad de emisión por medio de un modelo marxista clásico.

La economía es un sistema abierto, que intercambia flujos de energía y materia con el planeta. Todo proceso de transformación de materia orgánica en mercancías finales requiere el uso de energía. Con la mecanización de la producción, los combustibles fósiles asumieron un papel central en el capitalismo. Por ese motivo, la energía se incluyó en el modelo como un insumo, junto con el trabajo y el capital. Esto permitió analizar el crecimiento y el progreso técnico de manera más precisa. Se asumió la producción conjunta como caso general y se contextualizó la economía brasileña en un problema más amplio: la contaminación y la forma en que la acción humana afecta el proceso productivo, en una sociedad dependiente de los combustibles fósiles.

En la literatura marxista clásica se sugiere una serie de tendencias a largo plazo en las naciones capitalistas. A partir de lo observado en el caso del Brasil, se pueden enumerar los siguientes resultados: i) la producción del producto deseado y del producto no deseado aumentó durante el proceso de crecimiento económico del período 1970-2012; ii) la generación de PIB y las emisiones de $\mathrm{CO}_{2}$ aumentaron con el uso más intenso de trabajo, capital y energía; iii) la productividad del trabajo y la relación capital-trabajo crecieron, mientras la productividad del capital disminuyó y la productividad de la energía se mantuvo relativamente estable entre el primer y el último año, resultado coherente con la perspectiva marxista clásica; iv) el patrón dominante de progreso técnico encontrado fue de sesgo marxista y ahorrador de energía, caracterizado por el aumento de las tasas de crecimiento de la productividad del trabajo $\left(g_{x}>0\right)$, de la productividad de la energía $\left(g_{e}>0\right)$ y de la reducción de la tasa 
de crecimiento de la productividad del capital $\left(g_{\rho}<0\right)$; v); en el período en su conjunto, el crecimiento de la productividad dependió del aumento de la intensidad energética y de la relación capital-trabajo. Ese patrón es similar al observado en países en desarrollo. Sin embargo, es posible distinguir tres fases de progreso técnico en el Brasil en el período de referencia. Entre 1970 y 1980 se observó un patrón de sesgo marxista, seguido por un estancamiento técnico de 1980 a 2003. Por último, de 2003 a 2012, el patrón de progreso técnico fue de tipo neutral de Harrod.

Mientras que el PIB se distribuye entre los habitantes de un país, las emisiones de dióxido de carbono no se limitan a un único territorio. El producto no deseado se distribuye en la atmósfera, afectando en forma desigual a los habitantes del planeta y dificultando la coordinación política y económica para controlar su producción (Marquetti y Pichardo, 2013). La continuidad de ese patrón de creciente mecanización dependiente del uso intensivo de combustibles fósiles acelerará el proceso de cambio climático, que afectará negativamente a las futuras generaciones. La creciente acumulación de capital debe acompañarse con el aumento de la productividad de la energía, a fin de mitigar sus efectos negativos en el medio ambiente.

\section{Bibliografía}

Arrhenius, S. (1896), "On the influence of carbonic acid in the air upon temperature of the ground", Philosophical Magazine and Journal of Science, vol. 41, Londres.

Baran, P. y P. Sweezy (1966), Monopoly Capital: An Essay on the American Economic and Social Order, Nueva York, Monthly Review Press.

Boden, T., G. Marland y B. Andres (2010), Global, Fossil-fuel $\mathrm{CO}_{2}$ Emissions, Oak Ridge, Estados Unidos, Carbon Dioxide Information Analysis Center [en línea] http://cdiac.ornl.gov/trends/emis/tre_glob.html.

Burkett, P. (2003), "Capitalism, nature and the class struggle", Anti-capitalism: A Marxist Introduction, A. Saad-Filho (ed.), Londres, Pluto Press.

Callendar, G. S. (1938), "The artificial production of carbon dioxide and its influence on temperature", Quarterly Journal of the Royal Meteorological Society, vol. 64, №275, Royal Meteorological Society.

Carson, R. (1962), A primavera silenciosa, Lisboa, Editorial Pórtico.

Christiansen, J. (1976), "Marx and the falling rate of profit", American Economic Review, vol. 66, Nㅜ 2, Nashville, Tennessee, American Economic Association.

Daly, H. (1977), Steady-state Economics, San Francisco, Freeman.

Divisia, F. (1926), "L'indice monétaire et la théorie de la monnaie", Revue d'économie politique, vol. 40, № 1, París, Éditions Dalloz.

Duménil, G. y D. Levy (2003), "Technology and distribution: historical trajectories à la Marx", Journal of Economic Behavior \& Organization, vol. 52, № 2, Amsterdam, Elsevier.

(1995), "A stochastic model of technical change: an application to the US economy (1869-1989)", Metroeconomica, vol. 46, $\mathrm{N}^{\circ} 3$, Wiley.

Feijó, C. A. y M. T. Lamonica (2012), "Importancia del sector industrial para el desarrollo de la economía brasileña", Revista CEPAL, № 107 (LC/G.2536-P), Santiago, Comisión Económica para América Latina y el Caribe (CEPAL).

Foley, D. (2009), "The economic fundamentals of global warming", Twenty-first Century Macroeconomics: Responding to the Climate Challenge, J. Harris y N. Goodwin (eds.), Cheltenham, Edward Elgar. (1998), "Simulating long-run technical change", inédito.

Foley, D. y T. R. Michl (1999), Growth and Distribution, Cambridge, Harvard University Press.

Foster, J. B., B. Clark y R. York (2010), The Ecological Rift: Capitalism's War on the Earth, Nueva York, Monthly Review Press.

Furtado, A. y R. Carvalho (2005), "Padrões de intensidade tecnológica da indústria brasileira: um estudo comparativo com os países centrais", São Paulo em Perspectiva, vol. 19, № 1, São Paulo, Fundación SEADE.

Garegnani, P. y F. Petri (1989), "Marxismo e teoria econômica hoje", História do Marxismo, E. Hobsbawm (org.), vol. 12, Río de Janeiro, Paz e Terra.

Georgescu-Roegen, N. (1971), The Entropy Law and the Economic Process, Cambridge, Massachusetts, Harvard University Press. 
Harvey, D. (2006), The Limits to Capital, Nueva York, Verso.

Heston, A., R. Summers y B. Aten (2006), "Penn world table version 6.2", Center for International Comparisons of Production, Income and Prices, Universidad de Pennsylvania [en línea] http://pwt.econ.upenn.edu8.

IBGE (Instituto Brasileño de Geografía y Estadística) (2015), Sistema de Contas Nacionais. Brasil 2010-2013, Río de Janeiro, CD-Rom.

(2010), Sistema de Contas Nacionais. Brasil 2004-2008, Río de Janeiro, CD-Rom. (2003), Estatísticas do século XX, Río de Janeiro, CD-Rom.

_ (1990), Estatísticas históricas do Brasil: séries econômicas, demográficas e sociais de 1550 a 1988 , Río de Janeiro.

IPCC (Grupo Intergubernamental de Expertos sobre el Cambio Climático) (2007), Climate Change 2007: The Physical Science Basis, Cambridge, Cambridge University Press.

IPEA (Instituto de Investigación Económica Aplicada) (2016), "Ipeadata" [en línea] http://www.ipeadata.gov.br. Jacinto, P. A. y E. P. Ribeiro (2015), "Crescimento da produtividade no setor de serviços e da indústria no Brasil: dinâmica e heterogeneidade", Economia Aplicada, vol. 19, № 3, São Paulo, Universidad de São Paulo.

Jones, H. (1979), Modernas teorias do crescimento econômico: uma introdução, São Paulo, Atlas.

Kümmel, R. (1989), "Energy as a factor of production and entropy as a pollution indicator in macroeconomic modelling", Ecological Economics, vol. 1, № 2, Amsterdam, Elsevier.

Leontief, W. (1970), "Environmental repercussions and the economic structure: an input-output approach", The Review of Economics and Statistics, vol. 52, № 3, Cambridge, Massachusetts, The MIT Press.

Li, M. (2014), Peak Oil, Climate Change, and the Limits to China's Economic Growth, Nueva York, Routledge.

Marquetti, A. A. (2003), "A economia brasileira no capitalismo neoliberal: progresso técnico, distribuição de renda e mudança institucional", Anais do VIII Encontro Nacional de Economia Política, Florianópolis.

Marquetti, A. A. y G. Pichardo (2013), "Patterns of growth and technical change in the production of good and bad outputs", Investigación Económica, vol. 72, № 284, Ciudad de México, Universidad Nacional Autónoma de México (UNAM).

Marquetti, A. A. y M. Porsse (2014), "Patrones de progreso técnico en la economía brasileña, 1952-2008", Revista CEPAL, N 113 (LC/G.2614-P), Santiago, Comisión Económica para América Latina y el Caribe (CEPAL).

Marx, K. (1991), Capital, Londres, Penguin Books.

Maslin, M. (2004), Global Warming: A Very Short Introduction, Oxford, Oxford University Press.

MME (Ministerio de Minas y Energía) (2014), Balanço energético nacional, 2014, Brasilia. (2007), Balanço energético nacional, 2007, Brasilia.

Morishima, M. (1973), Marx's Economics: A Dual Theory of Value and Growth, Nueva York, Cambridge University Press.

Ocampo, J. A., C. Rada y L. Taylor (2009), Growth and Policy in Developing Countries: A Structuralist Approach, Nueva York, Columbia University Press.

Okishio, N. (1961), "Technical changes and the rate of profit", Kobe University Economic Review, № 7 , Universidad de Kobe.

Pérez, C. (2002), Technological Revolutions and Financial Capital: The Dynamics of Bubbles and Golden Ages, Northampton, Edward Elgar.

Pichardo, G. M. (2007), "Economic growth models and growth tendencies in major Latin American countries and in the United States, 1963-2003", Investigación Económica, vol. 66, Nㅜ 262, Ciudad de México, Universidad Nacional Autónoma de México (UNAM).

Roemer, J. (1977), "Technical change and the tendency of the rate of profit to fall", Journal of Economic Theory, vol. 16, № 2, Amsterdam, Elsevier.

Stern, N. (2006), Stern Review Report on the Economics of Climate Change, Londres, HM Treasury.

Taylor, L. (2008), "Energy productivity, labor productivity, and global warming", Twenty-first Century Macroeconomics: Responding to the Climate Challenge, J. Harris y N. Goodwin (eds.), Northampton, Massachusetts, Edward Elgar.

Vicente, M. (2014), "Influencia de la actividad económica en el ciclo natural del $\mathrm{CO}_{2}$ y su impacto en la temperatura terrestre", Tendencia y ciclo en economía: teoría y evidencia empírica, G. M. Pichardo (org.), Ciudad de México, Facultad de Economía, Universidad Nacional Autónoma de México (UNAM).

Vitousek, P. y otros (1997), "Human domination of Earth's ecosystems", Science, vol. 277, N 5325, Washington, D.C., American Association for the Advancement of Science (AAAS).

Von Arnim, R. y C. Rada (2011), "Labour productivity and energy use in a three-sector model: an application to Egypt", Development and Change, vol. 42, № 6, Wiley. 